NASA-CR-204703

A116S-3077

$N-92-\cdots$

\title{
STRUCTURE AND ROTATION OF THE SOLAR INTERIOR: INITIAI. RESULTS FROM THE MDI MEDIUM-L PROGRAM
}

\author{
A. G. KOSOVICHEV, J. SCHOU, P. H. SCHERRER, R. S. BOGART. R. I. BUSH, \\ I. T. HOEKSEMA. J. ALOISE, L. BACON, A. BURNETTE, C. DE FOREST, P. M. GILES, \\ K. LEIBRAND, R. NIGAM, M. RUBIN, K. SCOTT and S. D. WILLIAMS \\ W.W. Hanse'n Experimental Phusics Laboratory, \\ Stanford Universitu: Stanford. CA 94.3015, U.S.A. \\ SARBANI BASU and J. CHRISTENSEN-DALSGAARD \\ Theroretical Astrephysics Center, Danish National Rescarch Foundation, and lnstitute of Physics and \\ Astromomy, Aarhus Universiry, DK-SOOOO Aarhus C. Demmark \\ W. DÄPPEN and E. J. RHODES, JR. \\ Department of Physics and Astrmomy; Univerwity of Sonthem Califomia, Los Angeles. CA 90OS9. U.S.A. \\ T. L. DUVALL, JR. \\ Laboratory for Astronomy and Solar Phisics. \\ NASA Goddard Space Flight Center. (ireenbelt, MI) 2077 , U.S.A. \\ R. HOWE and M. J. THOMPSON \\ Astronomy Unit, Queen Mary and Westfield College. London, EI 4NS, U.K. \\ D. O. GOUGH and T. SEKII \\ Institute of Astronomy; and Department of Applied Mathematics and Theoretical Physics, Madingley Rocad. \\ Cambridge, $C B 3$ OHA, U.K. \\ J. TOOMRE \\ JILA. University of Colorade, CO 80309, U.S.A. \\ T. D. TARBELL. A. M. TITLE, D. MATHUR, M. MORRISON, J, L. R. SABA, \\ C. J. WOLFSON and I. ZAYER \\ Lockheed-Martin Adranced Technology Center \\ 91-30/252, 325/ Hanover St. Palo Alto, CA 94.304, U.S.A. \\ P. N. MILFORD \\ Parallel Rules, Inc: \\ 41 Manzanita Ave., Los Gatos, CA 9.5030, U.S.A.
}

(Received 23 October, 1996; in revised form 13 November, 1996)

\begin{abstract}
The medium-l program of the Michelson Doppler Imager instrument on board SOHO provide: continuous observations of oscillation modes of angular degree, $l$, from $0 t 0 \sim 300$. The data for the proggam are partly processed on board because only about $3 \%$ of MDI observations can be transmitted continuously to the ground. The on-board data processing, the main component of which is Gaussian-weighted binning, has been optimized to reduce the negative influence of spatial aliasing of the high-degree oscillation modes. The data processing is completed in a data analysis pipeline at the SOI Stanford Support Center to determine the mean multiplet frequencies and splitting coefficients.

The initial results show that the noise in the medium- $l$ oscillation power spectrum is substantially lower than in ground-based measurements. This enables us to detect lower amplitude modes and, thus, to extend the range of measured mode frequencies. This is important for inferring the Sun's internal structure and rotation. The MDI observations also reveal the asymmetry of oscillation spectral lines. The line asymmetries agree with the theory of mode excitation by acoustic sources localized in the upper convective houndary layer. The sound-speed profile inferred from the mean frequencies gives evidence for a sharp variation at the edge of the energy-generating core. The results also confirm the previous finding by the GONG (Gough ot al.. 1996) that, in a thin layer just beneath the convection zone, helium appears to be less abundant than predicted by
\end{abstract}

Solar Physics 170: 43-61, 1997.

(C) 1997 Kluwer Academic Publishers. Printed in Belgium. 
theory. Inverting the multiplet frequency splittings from MDI, we detect significant rotational shear in this thin layer. This layer is likely to be the place where the solar dynamo operates. In order to understand how the Sun works, it is extremely important to observe the evolution of this transition layer throughout the 11 -year activity cycle.

\section{Introduction}

Solar oscillations observed at the photosphere as fluctuations of Doppler velocity or intensity are usually described in terms of normal modes that are identified by three integers: angular degree $l$, angular order $m$, and radial order $n$. The frequencies of the normal modes depend on structures and flows in the regions where the modes propagate. These regions are different for different modes. In Figure 1 we show the spatial eigenfunction of a typical mode that propagates half way through the Sun in depth and at relatively low latitudes. The surface structure of the modes is represented by spherical harmonics, $Y_{l}^{m}(\theta, \phi)$. Because different modes sample different regions inside the Sun, by observing many modes one can, in principle, map the solar interior.

The principal goal of helioseismology is to infer the structure and dynamics of the Sun's interior from the oscillation frequencies of normal modes. The resolving power of these inferences depends on the accuracy of the frequency measurements, and on the frequency and angular degree bandwidths. Until now the frequencies of solar oscillations have been reliably measured only in a band around $3 \mathrm{mHz}$, from approximately 1.5 to $4.5 \mathrm{mHz}$, where the mode amplitudes peak. These oscillations are acoustic $(p)$ modes of low $l$ and high $n$, or of high $l$ and low $n$, and also fundamental $(f)$ modes of high $l$. Modes of low $l$ and low $n$ have not been observed from the ground because their amplitude is very small, much less than $1 \mathrm{~cm} \mathrm{~s}^{-1}$. Thus, one of the prime tasks of the MDI space experiment on board SOHO is to detect low-order modes. This requires long-term, continuous, stable observations free of atmospheric disturbances.

MDI has three basic helioseismology programs: medium- $l$, low- $l$, and dynamics (Scherrer et al., 1995). The regions of the Sun covered by these programs are shown in Figure 2. The first two programs have been run with almost no interruptions since 18 April, 1996. The medium-l data are spatial averages of the full-disk Doppler velocity out to $90 \%$ of the disk's radius measured each minute. This results in 26000 bins of approximately $10 \mathrm{arc} \mathrm{sec}$ resolution that provide sensitivity to solar $p$ modes up to $l=300$. The low-l observables are velocity and continuum intensity images summed into 180 bins, with the intent of detecting oscillations up to $l=20$. The dynamics program provides $1024 \times 1024$ images of the whole disk, thus covering all of the $p$ modes up to $l=1500$. However, the dynamics program can run continuously for only 2 months each year when the high-rate telemetry channel is available. Therefore, the frequency resolution of the dynamics data is somewhat limited. Also, the dynamics program cannot monitor the evolution of the solar structure and dynamics continuously. Therefore, it was important to extend the sensitivity of the medium-l program to as high a degree as possible. 


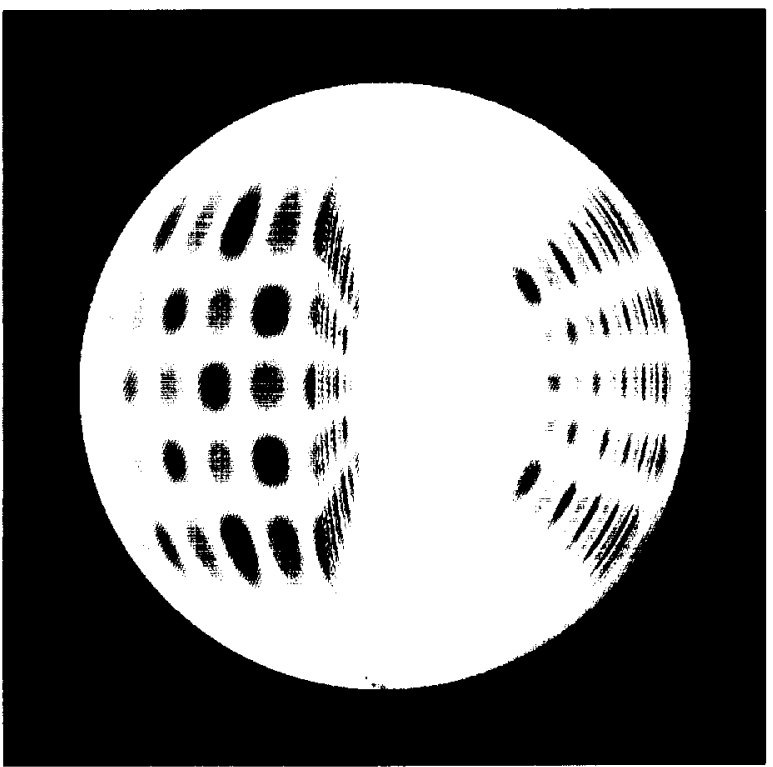

figme $l$. The spatial structure of the solar mode of angular degree $l=20$, angular order $m=16$, and radial order $n=14$. Red and blue show element displacements of opposite sign. The frequency of this mode determined from the MDI data is $2935.88 \pm 0.02 \mu \mathrm{H} \%$.

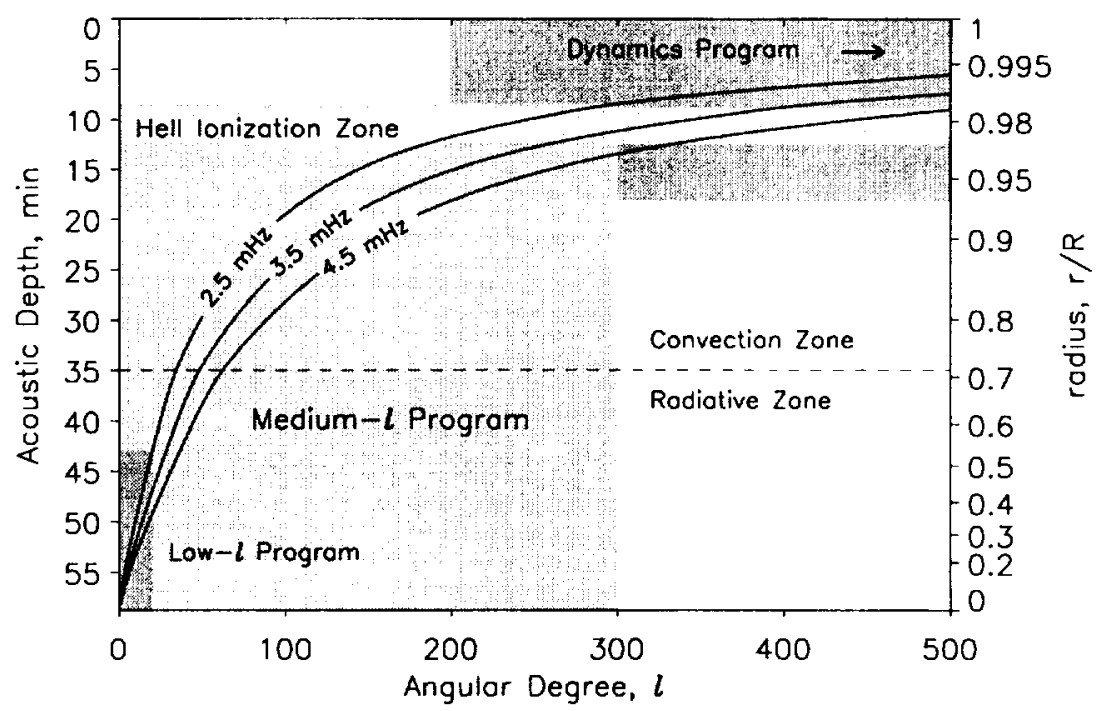

Figure 2. Acoustic depth and radius of penetration of solar modes of frequency of 2.5 .3 .5 , and $4.5 \mathrm{mH}$, as a function of angular degree, $l$ (black curves); and the areas covered by the MDI medium-l, low-l. and dynamics programs (shaded regions). The lower boundary of the convection rone is shown by the dashed line. The light-gray horizontal strip shows the location of the zone of the second ionization of helium. 
The target for the medium-l program is to observe the modes up to $l=300$. because these modes have their inner reflection points in the He Il ionization zone, and are, therefore. particularly sensitive to the structure of this zone. Studying the He ll ionization zone is important for measuring the helium abundance and calibrating the equation of state. and also for understanding the nature of supergranulation and the large-scale dynamics of the convection zone.

To optimize the medium-l observing program, we performed simulations of several vector-weighted binning schemes, using a 20-hour series of full-disk Dopplergrams obtained on 25 January, 1996. An optimal set of Galussian weights has been found that substantially reduces spatial aliasing in the angular degree range from 0 to 300 while preserving most of the power of the modes.

In Section 2, we describe the technique used in the medium-l program, the on-board data processing. and the basic parameters of the program. Analysis of the medium-l data is presented in Section 3. In Section 4, we discuss initial inversion results for the sound-speed profile and rotation.

\section{Medium-l Program Technique}

The medium-I data are transmitted through the low-rate telemetry channel (5 kilobits per second). The bit rate limits the spatial resolution of the observations. Prior to transmission the Doppler velocity is calculated from the original $1024 \times 1024$-pixel CCD filtergrams and binned on board the spacecraft. The binning can be performed in several ways depending on the scientific objectives of the program. For instance, Kosovichev (1992) considered spatially non-uniform binning schemes that maximize the range of the angular degree and maintain sensitivity to all the modes in this range. Alternatively, Gough (1992) suggested focusing on zonal and sectoral modes only, pushing the upper limit to $l=1500$ ). These and other possibilities will be explored in the course of the mission. However, the initial goal of the program is to obtain the most reliable measurements of modes of moderate that provide information about most of the interior of the Sun.

In observations performed with relatively low spatial resolution. the medium-l data can be corrupted because of spatial aliasing. Spatial aliasing contaminates normal mode peaks in the power spectrum with the signal from high-degree modes. This poses significant problems for measuring parameters of the modal lines. Therefore, we have adopted the Gaussian vector binning scheme suggested by Milford and Scherrer (1992). This reduces the spatial alialsing by averaging the data with Gaussian weights centered on a regular grid (saly of $4 \times 4$ - or $5 \times 5$-pixel spacing) on the $\mathrm{CCD}$ with a hall-width approximately equal to the spacing of the grid. Such a 2-D Gaussian filter also reduces solar noise. We have evaluated Gaussian-weighted binning using a 20-hour long time series of one-minute full disk $1024 \times 1024$-pixel Doppler images obtained during the MDI commissioning and initial calibration observations. 

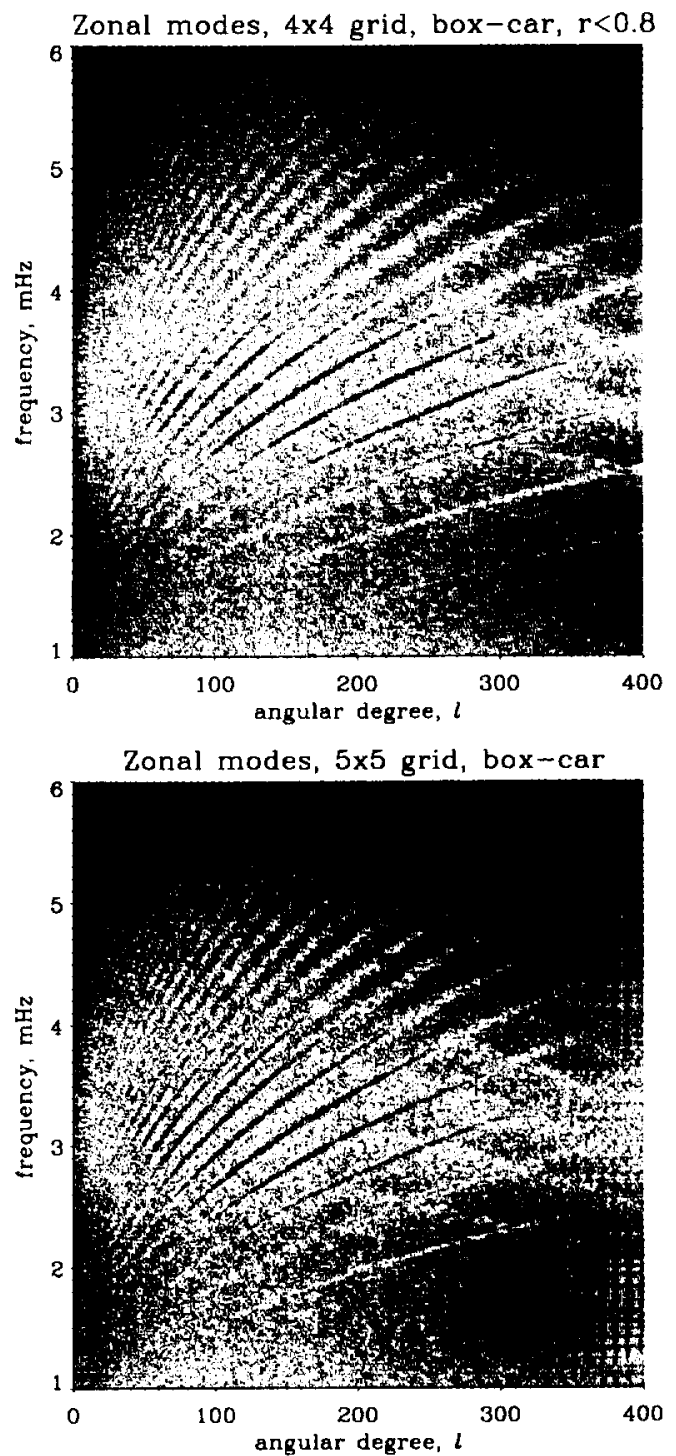

Figure 3. Power spectra of zonal modes computed using 20 hours of MDI full-resolution datil hox-call averaged onto a grid spacing of $4 \times 4$ pixels. covering the central part of the solat dish $(r<0.8 R)(t e p)$ and for a $5 \times 5$ grid spacing covering $90 \%$ of the disk (bomom).

\subsection{GAUSSIAN VECTOR BINNINGi}

For a vector binning scheme. the 2D filter function for a bin centered at the CCD pixel with coordinates $\left(i_{1} . j_{0}\right)$ takes the form

$$
W\left(i_{0}, j_{0} ; i, j\right)=u\left(i_{0} ; i\right) v\left(j_{0} ; j\right)
$$


Table I

Shot-noise reduction coefficient, $C_{n}$.

\begin{tabular}{lllllll}
\hline & $a=2.5$ & $a=3.5$ & $a=4$ & $a=4.5$ & $a=5.4$ & $a=6$ \\
\hline$c_{i 1}$ & 0.160 & 0.114 & 0.100 & 0.0189 & 0.074 & 0.066 \\
\hline
\end{tabular}

where $u\left(i_{0} ; i\right)$ and $v(j, j i)$ are functions of the CCD column number, $i$, and row number, $j$. respectively. While, in general, these functions can be arbitrary, the dyadic form of $W$ is essential for the on-board processing.

Following Milford and Scherrer (1992), we have represented $u$ and $v$ in the Gaussian form. i.c..

$$
u\left(i_{0} ; i\right)=W_{0} \exp \left[-\left(i-i_{0}\right)^{2} / a^{2}\right],
$$

and similarly for $\left.v_{(j)} ; j\right)$. Here, $a$ is the characteristic width of the Gaussians, and $W_{0}$ is a scaling factor. The centers $\left(i_{0}, j_{0}\right)$ of the bins were uniformly distributed on a $5 \times 5$-pixel rectangular grid in the area within 0.98 solar radii of disk center. The total number of bins was 29804. Results were evaluated for $a=2.5,3.5,4,4.5,5.4$ and 6 . For comparison we also computed oscillation power spectra for $5 \times 5$-pixel box-car averages over the full-disk and for $4 \times 4$-pixel box-car alverages in the central area restricted to $r<0.8 R$. Each case matches the telemetry bandwidth limit.

The data binning reduces the shot noise that determines the precision required in the on-board accumulations. We have estimated the coefficient of reduction of the shot noise, $C_{11}$, relative to the single pixel value as

$$
C_{n}=\frac{\sqrt{\sum_{i, j} W(i, j)^{2}}}{\sum_{i, j} W(i, j)} .
$$

The coefficients for the various values of $a$ are shown in Table $\mathbf{l}$.

\subsection{POWER SPECTRA OF THE SPATIALLY BINNED DATA}

Power spectrat of the binned data were computed using two different techniques: the standard method of remapping the images onto a rectangular grid of heliographic coordinates, and a method of direct projection on eigenmode masks (Kosovichev, 1992). The results obtained by both techniques are consistent with each other.

The power spectra of zonal modes $(m=0)$ computed with $4 \times 4$ and $5 \times 5$ box-car averaging are shown in Figure 3. Obviously, the data sampled on the finer spatial grid resolve $p$ modes at higher $l$ better. Also. the relative amplitudes of the aliased modes crossing the original mode ridges are substantially smaller. However, observations with $4 \times 4$-pixel averaging can only cover a smaller portion of the solar disk, increasing the spatial leakage from adjacent modes. This makes the mode ridges broader and complicates identification of individual oscillation mode frequencies. Therefore, for the initial observations, we require a $5 \times 5$-pixel grid which covers $90 \%$ of the full disk. 

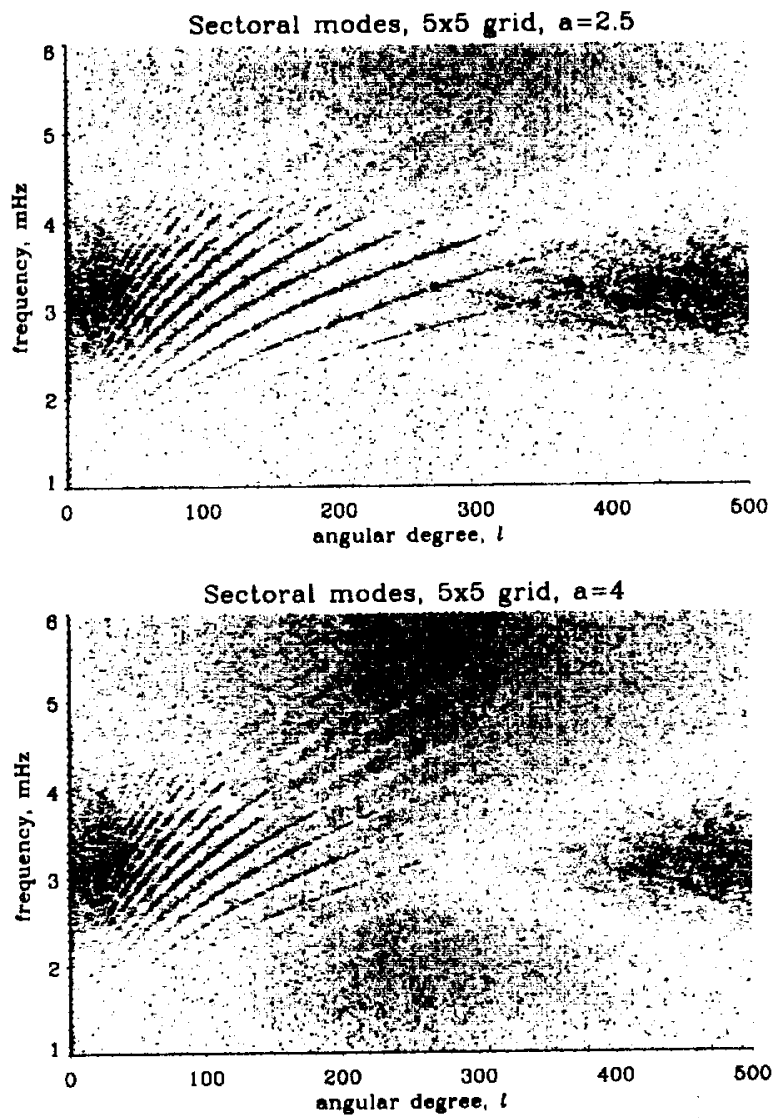

Sectoral modes, $5 \times 5$ grid, $a=5.4$

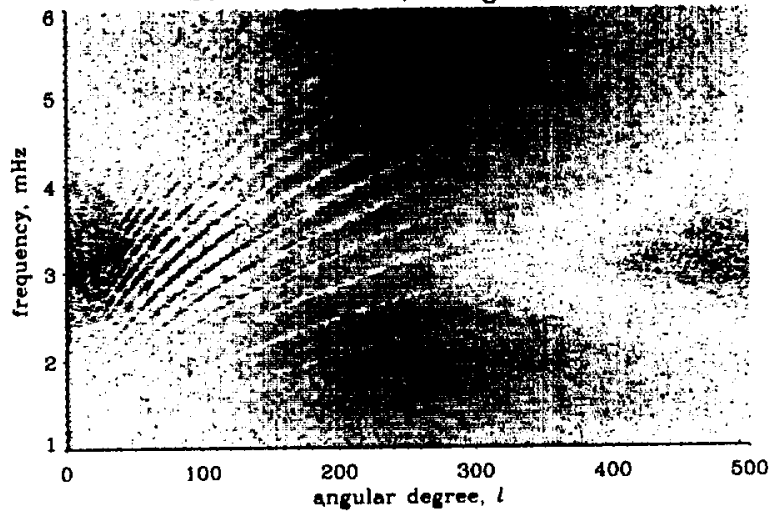

Figure 4. Power spectra of the sectoral modes obtained from 20 hours of the MDI full resolution spectrat using the Gaussian spatial filter of $a=2.5(t o p), 4($ middlle $)$, and $5.4(\mathrm{bon} / \mathrm{omm})$ 
Figure 4 shows the power spectra of the sectoral modes $(m=1)$ computed with Gaussian weighting with widths $a=2.5 .4$, and 5.4. As expected, increasing $a$ reduces the amplitudes of the aliased ridges, but it also reduces the amplitude of the original modes at higher $l$. For $a>4$, the amplitude of the 'true' modes in the range $l=200-300$ becomes too small. It seems that $a=4$ represents at reasonable tradeoff between anti-aliasing and high-l cut-off. In this case, the aliasing is substantially reduced up to $/=300$.

Therefore. for the initial medium-l program observations, we have chosen 10 use the Gaussian vector-weighted data binning on a rectangular grid of $5 \times 5$ pixels, in the form of Equations ( 1 ) and (2) with $a=4$. The shot noise of the binned data is reduced to 0.1 of the single-pixel value (Table I). If the noise per pixel per $60 \mathrm{~s}$ is $12 \mathrm{~ms}$ ' then the noise per bin is $1.2 \mathrm{~ms}$. The hinned images are generated on board once per minute.

\section{Analysis of the Medium-l Data}

\subsection{POWLR SPECTRA}

A standard helioseismic data analysis procedure (e.g.. Hill $e t$ al., 1996) has been applied to ohtain the oscillation power spectra from the medium- $l$ data. 60 days of data have been processed. The imalges were apodized with a cosine bell between $0.85 R$ and $0.89 R$ and remapped onto a grid with 768 points around the equator and 128 intervals between the ecpuator and pole. After this, they were Fourier transformed in longitude and multiplied by the associated Legendre functions $I_{I}^{\prime \prime \prime}(\theta)$ in colatitude $\theta$ to do the spherical harmonic decomposition.

The power spectrat of the sectoral and zonal modes, and also the m-averaged power spectrum. obtained from 10 days of the data are shown in Figure 5. The ridges in the diagrams correspond to modes of different radial order $n$. The lowest weak ridge is the $f$ mode. At low $l$. the ridges are not resolved in the plot of the $l$ - $/$ diagrams because the spectrum becomes too dense.

The alliasing effect is strongest for zonal $(m=0)$ and sectoral $(m=l)$ modes because these modes have the smallest spatial seale. It is clearly seen in the power spectrat of these modes for $l>220$. In the $m$-averaged spectrum (Figure 5, bottom), the effect is much weaker. It is important that aliasing of the fundamental modes, $f$, and first-order acoustic modes, $p_{1}$, be negligible up to $l=300$. This makes it possible to measure frequencies of individual modes along these two lowest ridges in the $l-l$ diagram. For higher p-mode overtones, individual modes are not resolved at $l>220$ beciuse the frequency difference between the modes of adjacent $l$ becomes smaller than the line widths of the modes. In this case. instead of measuring individual peaks, we plan to use 'ridge-fitting' techniques.

The medium-l program also provides good sensitivity to low-degree modes (Figure 6), thus covering almost the whole range of the solar oscillation spectrum, in which individual p) moder can be resolved. 

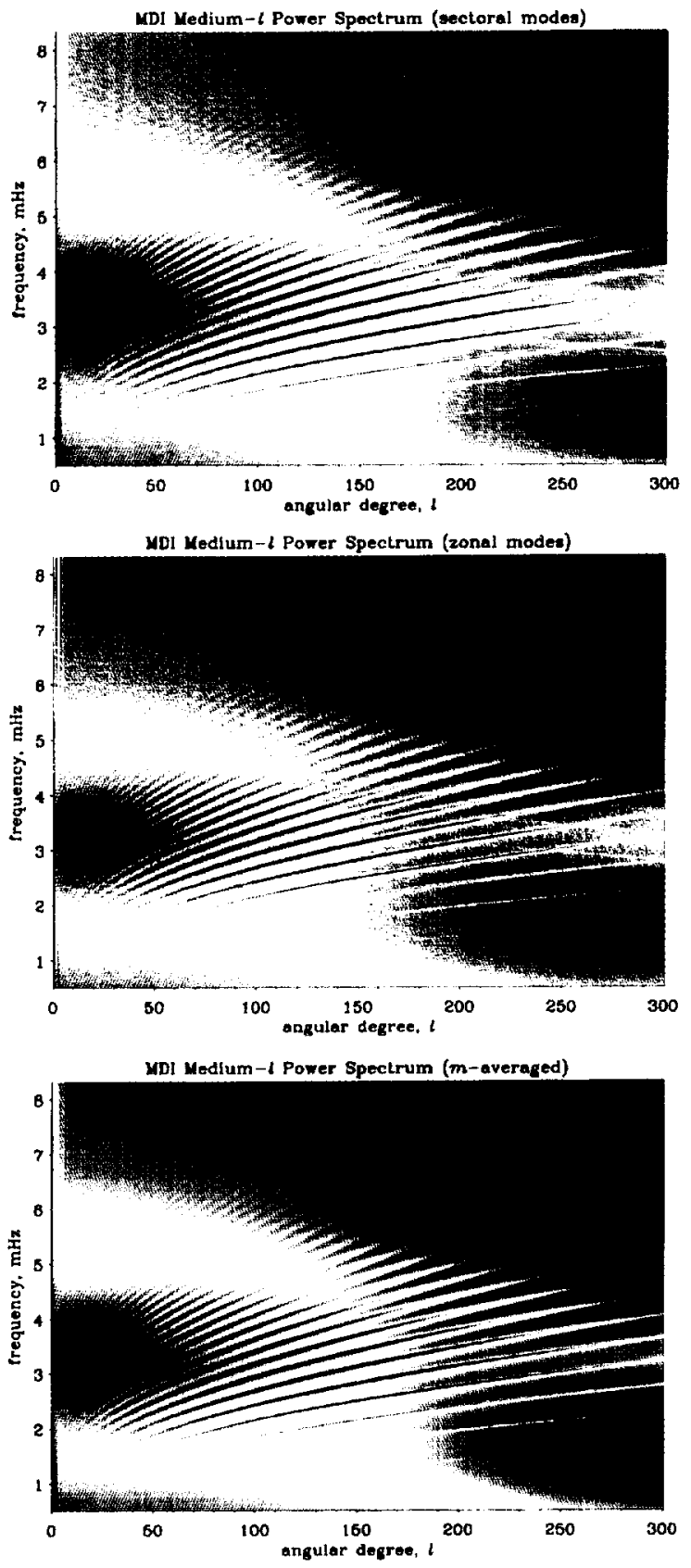

Figure 5. Power spectra (l- - $y$ diagrams) obtained from 10 days of the MDI medium-l data for the sectoral $(l=m)$ modes $(k o p)$, anal $(m=0)$ modes $($ middle). and for the modes averaged over $m$ (hottom). 


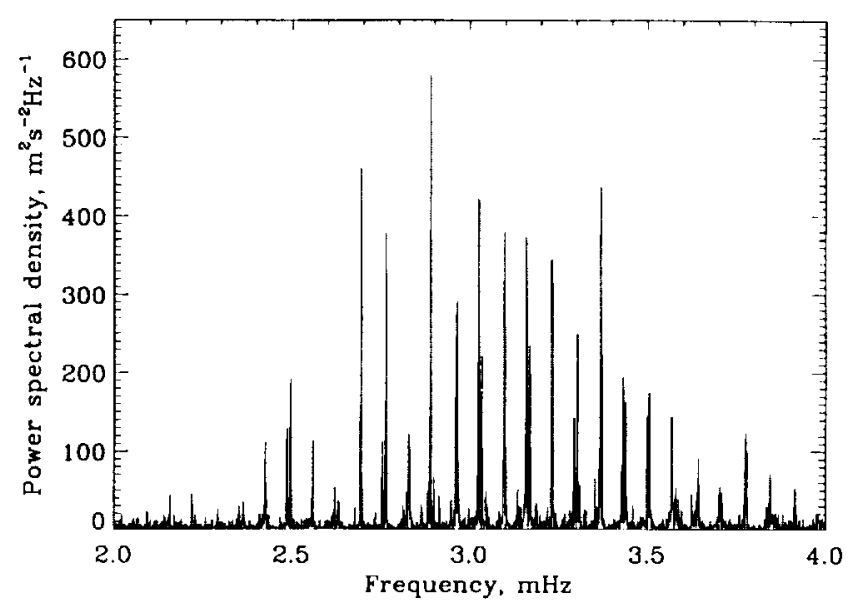

Figum o. Power spectrum of $l=0$. 1 , and 2 modes obtained from 10 days of the MDI dillat.

\subsection{RIESOLLITION OF LOW-FREQUENCY MODES}

An important difference between the MDI medium-l program and the ground-based networks is the ability to detect the low-n low-frequency modes that carry substantial independent information about the solar structure and dynamics. The lifetimes of these modes are much longer than the lifetimes of high-frequency modes. Therefore, in principle, the frequencies of these low-n modes can be determined more accurately than the frequencies of the other modes. However, the amplitude of the low-n modes is very small. so long stable time series are required to detect them. Two examples of low-frequency modes: the $p$ mode of $z=1$ and $l=50$, and the $f$ mode of $l=95$, are shown in Figures 7 and 8 . Parameters of these modes have been measured for the first time. With longer time series, we should be able to observe more low-frequency modes, with even lower amplitudes. So far, the smallest mode amplitude we have been able to detect was about $1 \mathrm{~mm} s$.

\subsection{LINE ASYMMETRY}

Becautse of the significant reduction in the noise, the medium- $l$ data have revealed interesting characteristics of the line profiles of the oscillation power spectra. Figure 9 shows the power spectra of the modes for $1=100,200$, and 300 . The most interesting fealure is the asymmetry of the line profiles. Though the asymmetry has been noticed in the groundbased data (Duvall et al. 1993), frequencies of solar modes are usually determined by assuming that the line profile is symmetric and can be fitted by a Lorentzian, which would be the case if the solar $p$ modes were damped simple harmonic oscillators excited by a stochastic source. However. this leads to systematic errors in the determination of frequencies (Hill et al. 1996; Abrams and Kumar, 1996). Several authors have studied this problem theoretically and have found that there is an inherent asymmetry whenever the 


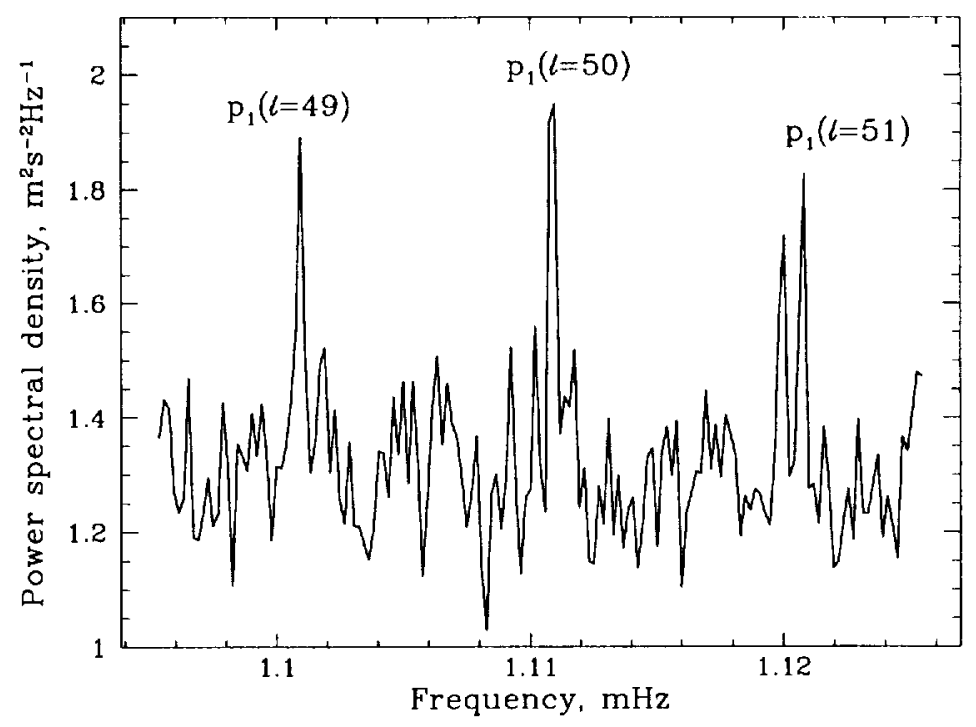

Figure 7. Power spectrum of $p$ mode of $l=50$ obtained from 2 months of the MDI medium-l data. Modes of adjacent $l$ leak into the spectrum because of imperfect spatial filtering.

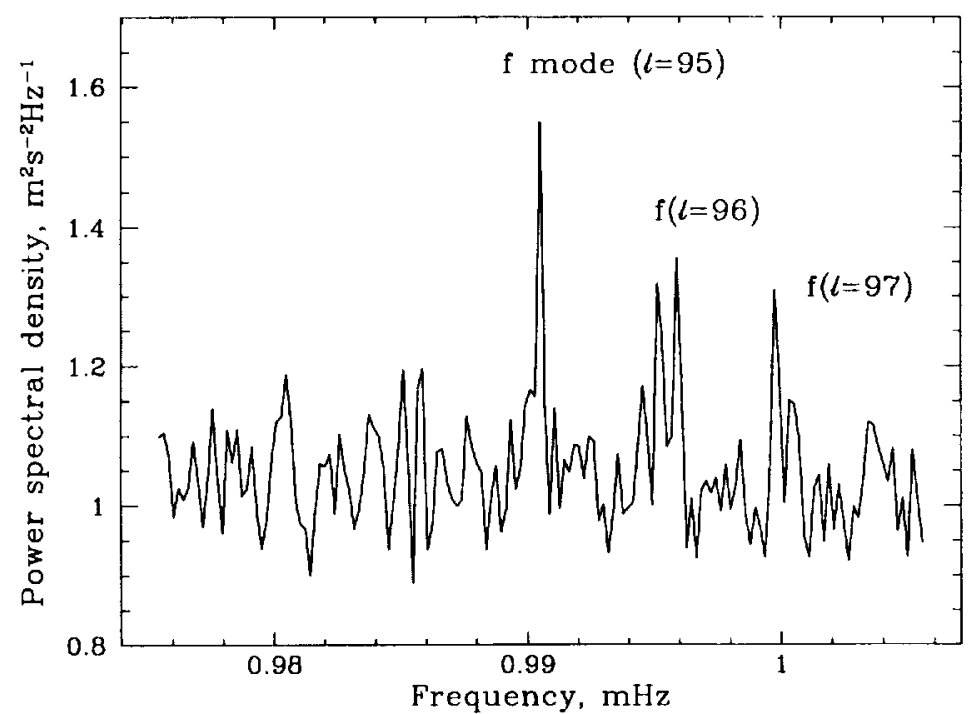

Figure 8 . Power spectrum of $f$ mode of $l=95$ obtained from 2 months of the MDI dita.

waves are excited by a localized source (Gabriel, 1992, 1993, and 1995; Kumar et al. 1994; Roxbourgh and Vorontsov, 1995). Physically, the asymmetry is an effect of interference between an outward direct wave from the source and a corresponding inward wave that passes through the region of wave propagation (Duvall et al., 1993). Figure 10 shows a theoretical power spectrum of $p$ modes of $l=200$ obtained by Nigam and Kosovichev 

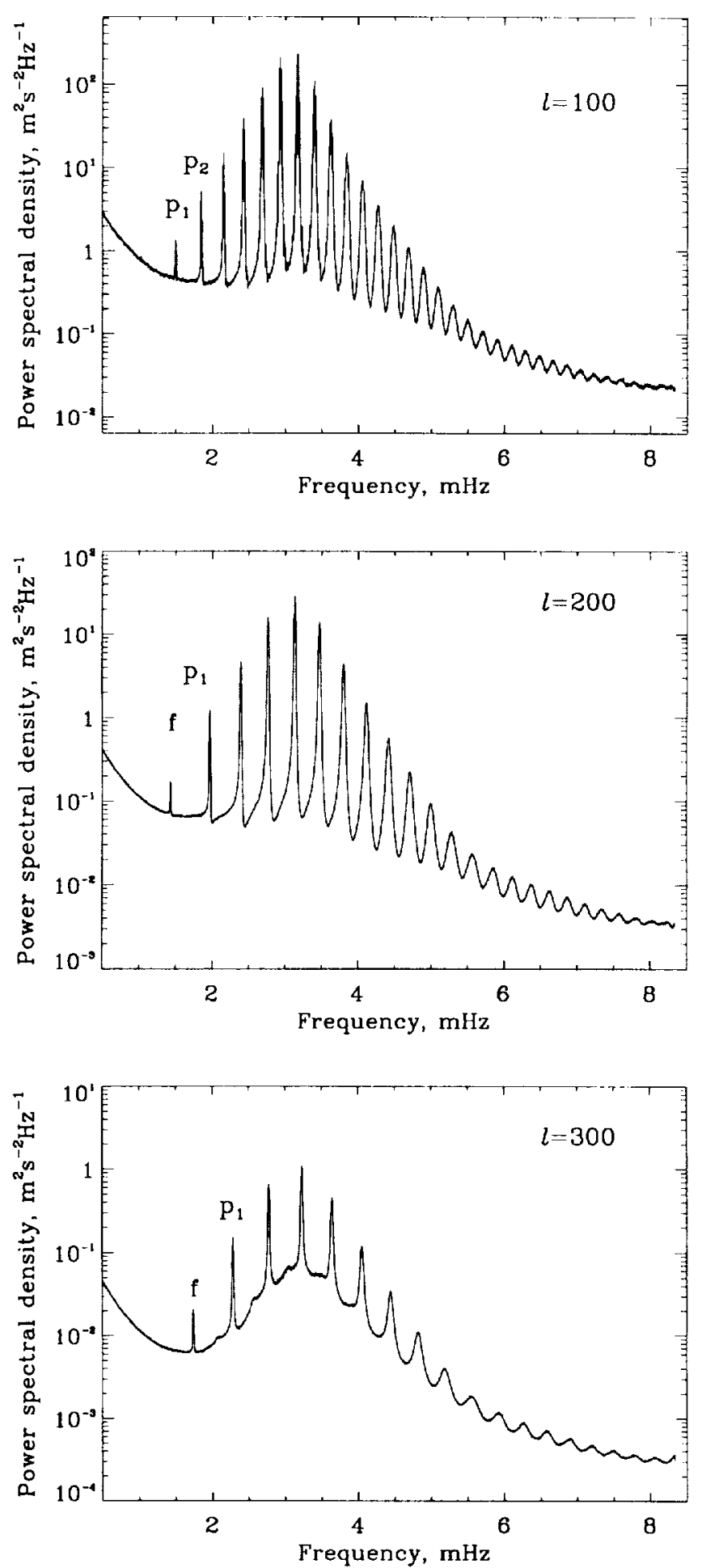

Figlure 9. Power spectra obtained from 10 days of the MDI medium- $l$ data of modes of $l=100(t o p), l=20 \%$ (middll'), and $l=3(K)($ bottom). Small bumps between the main peaks in the $l=300$ spectrum are spattial alialses (sece Section 3.2) 


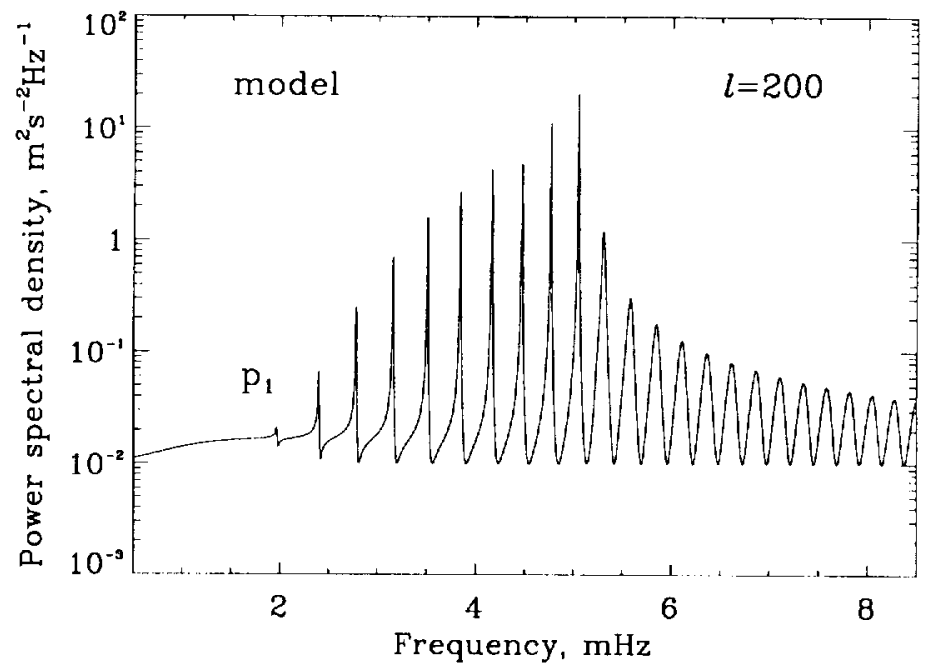

Figure 10. Theoretical power spectrum of $l=2(0)$ mode for a model with a source of the uniform spectral density localized $80 \mathrm{~km}$ beneath the photosphere. The background noise is assumed to be uniform with the power spectral density $10^{-2} \mathrm{~m}^{2} \mathrm{~s}^{-2} \mathrm{H} /{ }^{\prime}$.

(1996). This model is in good qualitative agreement with the observations. The degree of the asymmetry depends on the relative locations of the acoustic sources and the upper reflection layer of the modes. This opens the prospect of using the observations of the line profiles of solar modes to test theories of excitation of solar and stellar oscillations and of their interaction with turbulent convection.

\subsection{FREQUENCY MEASUREMENTS}

The frequencies of the solar oscillations have been measured as described by Schou (1992). The frequency splitting of mode multiplets due to rotation and asphericity is approximated using a polynomial expansion similar to that of Duvall. Harvey and Pomerantz (1986):

$$
\nu_{n l m}=\nu_{n l}+\sum_{k=1}^{6} a_{n l}^{(k)} \mathcal{P}_{k}^{(l)}(m),
$$

where $\nu_{n l}$ is the mean frequency of a mode multiplet, and $\mathcal{P}_{k}^{(\prime)}(m)$ are orthogonal polynomials of degree $k$ defined by

$$
\mathcal{P}_{k}^{(l)}(l)=l, \quad \text { and } \quad \sum_{m=-l}^{l} \mathcal{P}_{i}^{(l)}(m) \mathcal{P}_{j}^{(l)}(m)=0 \quad \text { for } \quad i \neq j
$$

The polynomials, $\mathcal{P}_{k}^{(l)}(m)$, can be expressed in terms of the Clebsch-Gordan coefficients, $C_{k \cdot 1: m,}^{l m}$,

$$
\mathcal{P}_{k}^{(l)}(m)=\frac{l \sqrt{(2 l-k) !(2 l+k+1) !}}{(2 l) ! \sqrt{2 l+1}} C_{k \cdot(l) l m}^{l m} .
$$




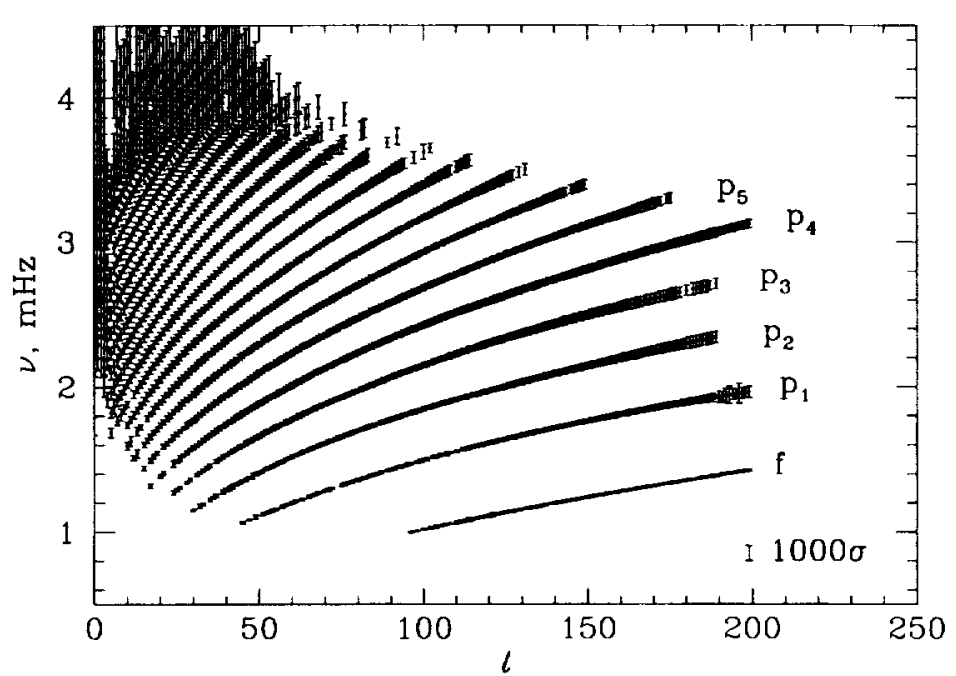

Figure /I. Mean frequencies of the mode multiplets obtained from 2 months of the medium-l dati. The error bars show the standard errors multiplied by 1000 .

Since

$$
\sum_{m=1}^{1} P_{k}^{(l)}(m)=0 \text { for } k>0 .
$$

the mean frequencies, $\nu_{n}$. depend only on the spherically-symmetric component of the solar structure. Frequency splitting coefficients, $a_{m l}^{(k)}$, for even $k$ depend on the aspherical component of the structure, while the coefficients for odd $k$ measure the rotation rate (e.g.. Gough, 1993).

For the initial frequency measurements, we approximated the line profiles by Lorentzians and used a maximum likelihood method to determine the parameters of the Lorentzians, taking into account possible overlaps of lines. The effect of the line asymmetry wass not taken into account in these initial measurements. The mean frequencies of mode multiplets are shown in Figure 11. The error bars in this figure indicate estimated errors multiplied by 1000 .

\section{Initial Inversion Results}

\subsection{RADIAL. STRATIFICATION}

We have determined the spherically-symmetric structure of the Sun by using the optimally localized averaging techniques (e.g., Gough, 1985; Pijpers and Thompson, 1992) to invert the mean frequencies of split mode multiplets, $\nu_{n l}$. Figure 12 shows the relative difference between the square of the sound speed in the Sun and a reference solar model. The 


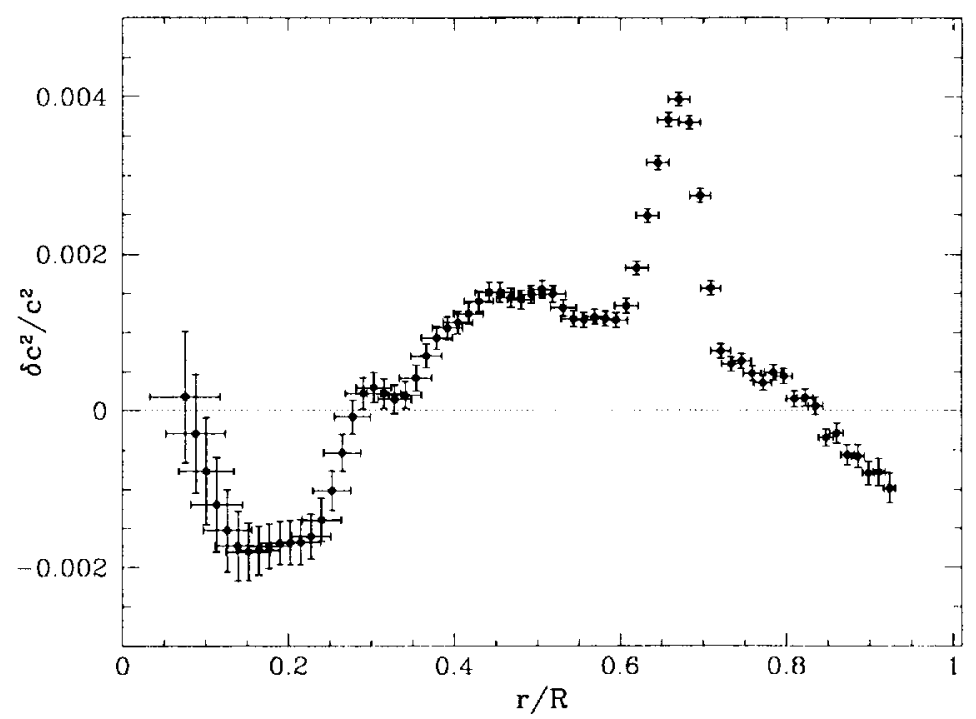

Figure 12. Relative differences hetween the squared sound speed in the Sun and a standard solar model as inferred from 2 months of the MDI data. The horizontal bars show the spatial resolution. and the vertical bars are error estimattes.

reference model was model $S$ of Christensen-Dalsgaard et al. (1996). This model was a standard evolutionary model computed using the most recent information on nuclear reaction rates (Bahcall and Pinsonneault, 1995) and radiative opacity and the equation of state (Rogers and Iglesias, 1996). The gravitational settling and diffusion of helium and heavier elements were taken into account following the theory by Michaud and Proffitt (1993).

The inversion results show that the maximum difference in the square of the sound speed between the model and the Sun is only $0.4 \%$. Nevertheless, this difference is very important for understanding solar evolution and physical processes inside the Sun. Two features of the sound-speed profile are particularly notable. The first is a narrow peak centered at $0.67 R$, just beneath the convection zone. This peak was previously detected in the LOWL (Basu et al., 1996) and GONG data (Gough et al., 1996) and is most likely due to a deficit of helium in this narrow region. The deficit of helium decreases the mean molecular weight and thus increases the sound speed. The deficit of helium could result from additional mixing of the material in the layer with the surrounding plasma if turbulence is generated in this layer because of rotational shear. Indeed, as we show in the next section, there is a strong radial gradient of the rotation rate in this layer.

Another interesting feature is the sharp decrease of the sound speed compared to the model at the boundary of the energy-generating core, at $0.25 \mathrm{R}$. This variation is not explained by the theory. Kosovichev and Fedorova (1991) noticed a possible sharp increase of density at the edge of the core when inverting the BBSO datal (Libbrecht, Woodard, and Kaufman, 1990) if the spatial resolution of the inversion was increased at the expense of substantially increased errors of the estimates. The evidence from the MDI 
data is more convincing. It is quite possible that the drop in the sound speed results from an overabundance of helium at the edge of the solar core.

The steep increase of the sound speed towards the solar center can be explained if helium is less abundant than in the standard solar model. This indicates that the helium abundance profile seems to be more flat in the solar core than it is in the standard solar model. Gough et al. (1996) came to a similar conclusion from the GONG data. In principle, this variation of the helium abundance could arise from errors in the rates of the nuclear reactions or element diffusion. It could also be explained by uncertainties in the equation of state and in radiative opacity. Helioseismology is unable to distinguish among the possibilitics without additional assumptions about the physical processes. However, if the transition at the edge of the core is really as sharp as we have found from the initial MDI data, then it strongly suggests material redistribution by macroscopic motions in the core, possibly induced by the instability of ${ }^{3} \mathrm{He}$ burning, as first suggested by Dilke and Gough (1972). Detailed studies of this phenomenon are very important for understanding stellar evolution. The deviations from the standard model of the spherically averaged structure of the solar core are fairly small; too small to solve the solar neutrino problem directly. However, if an instability really occurred, as predicted by theory (ChristensenDalsgaard, Dilke, and Gough, 1974; Boury et al. 1975; Shibahashi, Osaki and Unno, 1975: Kosovichev and Severny, 1985: Merryfield, Toomre and Gough, 1991), then the basic assumptions of solar evolution theory about the thermal balance in the core must be questioned. This is certainly important for understanding the nature of the solar neutrino problem and for using the Sun as a laboratory for particle physics.

\subsection{INTERNAL ROTATION}

The internal rotation rate at three latitudes, 0 (equator), 30 and 60) deg, is shown in Figure 13. The rotation is inferred from the odd $a_{n l}^{(k)}$ coefficients of Equation (3) using a regularized least-squares technique (e.g., Tikhonov and Arsenin, 1977). The inversion results confirm the previous findings that latitudinal differential rotation occurs only in the convection zone, that the radiative interior rotates almost rigidly, and that there is a thin shear layer near the surface (Goode et al., 1991: Thompson et al., 1996). The most interesting feature is that the transition layer (tachocline) between the radiative and convection zone is mostly located in the radiative zone. at least at the equator where it is also fairly thin, certainly less than $0.1 R$. The layer seems to be wider at high latitudes. Previously, the parameters of the tachocline were estimated by Kosovichev (1996) and by Charbonneau et al. (1996) by fitting an analytical model to the data from the BBSO (Woodard and Libbrecht, 1993), and from the LOWL instrument (Tomezyk et al., 1995).

The sharp radial gradient of the angular velocity beneath the convection zone strongly suggests that the sharp narrow peak of the sound speed at $0.67 R$ seen in the structure inversion (Figure 12) is indeed due to rotationally-induced turbulent mixing in the tachocline (Spiegel and Zahn, 1992). The location and the width of the tachocline are also consistent with the requirement of recent dynamo theories of the solar cycle (Rüdiger and Brandenburg, 1995; Weiss, 1994). It will be extremely important to observe the variations 


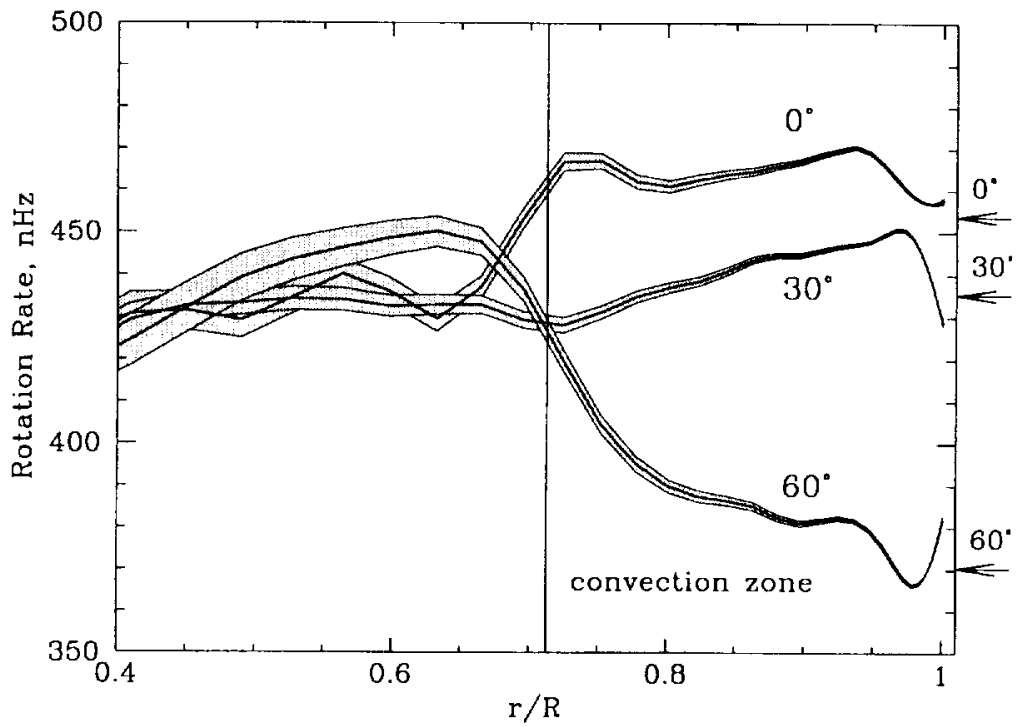

Figure 13. Solar rotation rate inferred from 2 months of MDI medium-l data as a function of radius at three latitudes, $0^{\circ}, 30^{\circ}$, and $60^{\circ}$. The formal errors ate indicated by the shaded regions. The arrows indicate the Doppler rotation rate directly measured on the surface.

of the angular velocity in the tachocline through the solar cycle in order to understand the mechanism of solar activity.

\section{Conclusions and Perspectives}

The MDI medium- $l$ program is designed for continuous monitoring of the structure and dynamics of the Sun's interior. The initial results show that the noise in the medium-l oscillation power spectrum is substantially lower than in the ground-based measurements. This enables us to resolve lower amplitude modes and, thus, to extend the range of measured mode frequencies. This is important for inferring the Sun's internal structure and rotation. The MDI observations clearly show the asymmetry of oscillation line profiles in agreement with the theory of mode excitation by acoustic sources localized in the upper convective boundary layer. The sound-speed profile inferred from the mean frequencies gives evidence for a sharp variation at the edge of the energy-generating core, which is not accounted for by the standard evolution theory. The results also confirm the previous finding from GONG data that helium appears to be less abundant than predicted by theory in a thin layer just beneath the convection zone (Gough et al., 1996). Inverting the multiplet frequency splittings from MDI, we have found significant rotational shear in this thin layer. This shear flow probably generates turbulence that mixes the plasma in the upper radiative zone. This layer ('tachocline') is likely to be the place where the solar dynamo operates. 
The first results of the medium-l program suggest the initial binning scheme should be maintained for the first year of SOHO observations to obtain the time series sufficient to resolve low-frequency $p$ modes. After the first year, it may be interesting to explore other possibilities, such as decreasing the remaining aliasing for modes of $l=200-300$ to allow identification of at greater number of modes, or using an inhomogeneous binning scheme to focus on some particular groups of modes. Certainly, the decision should be taken after consideration of the performance of the other $\mathrm{SOHO}$ and ground-based helioseismic programs. However, the principal goal of the medium-l program will remain the same continuous measurement of the solar structure and dynamics as they change through the solar cycle. This is crucial for understanding the mechanisms for solar variability.

\section{Acknowledgements}

We thank John Leibacher for commenting, as referee, on the original submitted version of this paper.

The authors acknowledge many years of effort by the engineering and support staff of the MDI development team at the Lockheed Palo Alto Research Laboratory (now Lockheed-Martin) and the SOI development team at Stanford University. SOHO is a project of international cooperation between ESA and NASA. This research is supported by the SOI-MDI NASA contract NAG5-3077 at Stanford University.

\section{References}

Abrams, D. and Kumar, P.: 1996, Astrophss. J. 472.882

Bahcall. J. N. and Pinsonneault. M. H.: 1995. Re1: Mod. Phys. 67, 781.

Bast. S.. Christensen-Dalsgatrd, J.. Schou, J., Thompson. M. J., and Tomeryk, S.: 1996. Bull. Astr: Soc. India 24. 147.

Boury, A.. Giabriel, M. Noels, A., Scuflaire, R., and Ledoux, P.: 1975, Astron. Astrophys. 41. 279.

Brandenture. A.: 1994, in M. R. E. Proctor and A. D. Gilbert (eds.). Lectures on Solar and Planetary Dynamo, Cambridge University Press. Cambridge, p. 177.

Charbonneau. P., Christensen-Dalsgatard. J., Henning. R.. Schou. J.. Thompson, M. J., and Tomczyk, S.: 1996, in J. Provost and $\mathrm{E}: \mathrm{X}$. Schmider (eds.). Sorunding Solar and Stellar Interiors, Proce. IAU Simp). 181, in press.

Christensen-Dalsgaard. J.. Dilke, F. W. W. and Gough, D. (). 1974. Monthl Notices Ros: Astron. Sor. 169. 429.

Christensen-Dalsgatard, J., Gough, D. O., and Thompsom, M. J.: 1991. Astrophss. J. 378. 413.

Christensen-Datilsgard, J.. Dïppen. W., and the GONG Team: 1996. Science 272, 1286.

Dilke. F. W. W. and Gough, D. O.: 1972. Nature 240. 262.

Duvall. T. L.. Halrvey. J. W. and Pomeranty. M. A.: 1986, Nature 321, 500.

Duvall. T. I... Jr., Jefferies, S. M., Harvey, J. W.. Osaki, Y., and Pomerantr. M. A.: 1993, Astrophys. J. 410. 829.

Giabricl. M.: 1992. Astron. Astrophess. 265. 771.

Githricl. M.: 1993, Astron. Astrophis. 274, 935

Gituriel, M.: 1995, Astron. Astrophys. 299, 245.

Gorkle. P. R.. Driembowski, W. A., Kornennik. S. G., and Rhodes, E.J.: 1991. Astrophys. J. 367, 649.

Gough, D. ().: 1985, Solar Phws. 100, 6.5.

(iongh. D. ().: 1992. On POX Strmeg!. SOL-TN-(099. Stantord University. 
Gough, D. O.: 1993, in J. P. Zahn and J. Zinn-Justin (eds.). Astrophesical F/hid Dinamics. Elsevier, Amsterdam, p. 399.

Gough, D. O., Kosovicher, A. G., and the GONG Team: 1996, Scicher 272, 1296.

Hill. F. Statk. P. B., Stebbins. R. T., and the GONG Team: 1996, Science 272. 1292.

Kosovichev. A. G. and Fedorova, A. V.: 1991, Astron. Zh. 68. 1051.

Kosovichev, A. G. and Severny, A. B.: 1985, In: Krimsk. Astrofic. Obs. $72,188$.

Kosovichev, A. G.: 1992, Optimal Masks for Strmiture Program, SOI-TN-096, Stanford University.

Kumar, P., Fandal, M. A., Jefferies, S. M., Duvall, T. L., Jr., Harvey, J. W., and Pomerant\%. M. A.: 1994. Astrophes. I. 422, L29.

Libbrecht, K. G., Woodard, M. F., and Kaufman, J. M.: 1990, Astmphss. J. Sitppl. 74. 1129.

Merryfield. W. J., Toomre, J.. and Gough. D. ( .; 1991, Astrophs. J. 367, 658.

Milford. P. N. and Scherrer, P. H.: 1992. Omline of Gamssiam Mask Mode, SOI-TN-058, Stanford University.

Michaud, G. and Proftitl. C. R.: 1993, in A. Baglin and W. Weiss (eds.), Inside the Stars, ASP Conf. Series. Vol. 40. San Francisco, p. 246.

Nigam. R. and Kosovichev, A. G.: 1996, in J. Prowost and F. X. Schmider (eds, ). Sounding Solar and Stellar Interiors: Proc: AU Simp. 181, in press.

Pijpers, F. P. and Thompsom, M. J.: 1992, Astrm. Astrophss. 262, L33.

Rogers, F. J. and Iglesias, C. A.: 1996, Astrophys. J. 456, 902.

Roxbourgh, 1. W. and Vorontsow, S. V.: 1995, Momthly Notices Ren. Astron. Soc. 272. 850).

Riidiger, G. and Brandenhurg, A.: 1995, Astrm. Astrophys. 296. 557.

Silio. H.: 1980, Astrophs. J. 240. 685.

Scherrer. P. H., Bogart. R. S., Bush. R. I., Hocksema, J. T., Kosovichev, A. G., Schou, J., Rosenberg, W., Springer, L., Trabell, T. D., Tille, A., Wolfson, C. J., Zayer, I., and the MDI Engineering Team: 1995 , Solar Phys. 162, 129.

Schou, J.: 1992, 'On the Analysis of Helioseismic Dala", Thesis, Aarhus University.

Shibahashi, H., Osaki, Y., and Unno, W.: 1975, Publ. Astron. Soc. Kapan 27, 401.

Spiegel, E. A. and Zahnn, J.-P.: 1992, Astom. Astrophlys. 265. 106.

Thompson, M. J., Toomre, J., and the GONG Team: 1996, Sicience 272, 1.300.

Tikhonov, A. N. and Arsenin, V. Y.: 1977. Solution of IIt-posed Problems, Winston, Washington. DC.

Tomeryk, S.. Streander, K.. Card, G, Elmore, D., Hull, H., and Cacciani, A.: 1995. Solar Phys. 159, 1.

Weiss, N. O.: 1994, in M. R. E. Proctor and A. D. Gilbert (eds.), Lectures on Solar and Planetary Dymame, Cambridge University Press. Cambridge, p. 59.

Woodard, M. F. and Libbrech1. K. G.: 1993, Srience 260. 1778. 
